\title{
Hiponatremia secundaria a diuréticos
}

\author{
J. Las Heras M osteiro, M. Taboada TABoada* \\ Médico Residente de Mediana Familiar y Comunitaria. \\ * Especialista en Cardiología. Centro de Salud Fuencarral. Madrid
}

\section{Secondary hyponatremia caused by diuretics}

\section{RESUMEN}

La hiponatremia secundaria al tratamiento diu rético es la primera causa de hiponatremias en los adultos. La correcta utilización de los diuréticos por el Médico de Familia requerirá un conoci miento de la farmacología de cada grupo así como de la fisiopatología de la enfermedad de cada paciente.

El caso clínico que presentamos a continuación trata de una mujer de 70 años, con insuficiencia cardiaca grado funcional III-IV de la NYHA, se cundaria a miocardiopatía multivalvular. Con un marcapasos VVI Medtronic por enfermedad del seno sintomática. En tratamiento con furosemida, espironolactona, enalapril, digoxina y anticoagu lantes orales. Presentó un cuadro de desorienta ción y desconexión del medio, objetivando en la analítica una hiponatremia severa.

Palabras clave: Diuréticos. Hiponatremia. Efectos adversos.

\begin{abstract}
The most important cause of hyponatremia in adults is diuretic's therapy. Effective use of diure tics by the family doctor, requires knowledge of the pharmacology of each diuretic agent coupled with an understanding of pathophysiology of the pa tient's disease.

We report a case of 70-years old female who had heart failure class III-IV New York Heart Associa tion (NYHA) secondary to multivalvular cardiom yopathy. She had implanted VVI Medtronic pace maker for sick-sinus syndrome. Treatment with furosemide, spironolactone, enalapril, digoxine and oral anticoagulant. She presented a clinical pattern of disorientation and a swich-off episode with severe hyponatremia.
\end{abstract}

Key words: Diuretics. Hyponatremia. Adverse effects.

\section{INTRODUCCIÓN}

Las causas más frecuentes de hiponatremia severa en adultos son: el tratamiento diurético con tiazidas, los estados postoperatorios, síndrome de secreción inapropiada de hormona antidiurética, polidipsia en pacientes psiquiátricos y prostatectomía transuretral ${ }^{1}$.
El tratamiento diurético está presente en más de la mitad de las hospitalizaciones por hiponatremia crónica grave, y afecta sobre todo a pacientes ancianos. La hiponatremia es más frecuente en las mujeres que en los hombres. Las mujeres de edad avanzada y bajo peso son muy susceptibles a la hiponatremia inducida por diuréticos, debido a que para producir ese efecto sólo son necesarias en 
ellas ganancias pequeñas de agua y pérdidas pequeñas de $\mathrm{sal}^{2}$.

Aunque la hiponatremia se encuentra más relacionada con el uso de los diuréticos tiazídicos, la adicción de un diurético de asa a una tiazida favorece el efecto natriurético. Por otra parte, los diuréticos ahorradores de potasio pueden exacerbar el desarrollo de la hiponatremia ${ }^{3}$.

La hiponatremia hipotónica genera movimiento de agua hacia las células, con tumefacción celular. La tumefacción de las células encefálicas origina edema cerebral que conduce a los síntomas neurológicos de la hiponatremia: cefalea, náuseas, vómitos, calambres musculares, letargia, sedación, desorientación y reflejos disminuidos. Los síntomas de la hiponatremia guardan una estrecha correlación con la intensidad y rapidez del descenso de las concentraciones de sodio ${ }^{1}$.

Presentamos un caso clínico de una paciente con insuficiencia cardiaca grado funcional IIIIV de la NYHA que presentó un cuadro de hiponatremia severa secundario al tratamiento diurético.

\section{CASO CLÍNICO}

Se trata de una mujer de 70 años y $35 \mathrm{~kg}$ de peso, seguida en nuestra consulta de Atención Primaria y en el Servicio de Cardiología de la Clínica Puerta de Hierro de Madrid. Como antecedentes personales, la paciente no tenía hábitos tóxicos, no era hipertensa, ni tenía dislipemias. La paciente tenía insuficiencia cardiaca secundaria a miocardiopatía multivalvular, siendo portadora de una doble prótesis de Björk aórtica y mitral con anuloplastia tricuspídea desde 1983, con grado funcional III-IV de la NYHA. Así mismo tenía implantado en zona epicárdica un marcapasos VVI Medtronic por enfermedad del seno sintomática, desde 1991. Como enfermedades no cardiológicas tenía una leucotrombopenia secundaria a hepatopatía crónica por VHC. Se encontraba en tratamiento con digoxina, acenocumarol y furosemida.

Acude a urgencias por aumento progresivo de su disnea habitual hasta hacerse de reposo, ortopnea de 2 almohadas y aumento progresivo de sus edemas en miembros inferiores (MMII), es diagnosticada de insuficiencia cardiaca descompensada por disfunción del marcapasos, recambiando el generador y dándola de alta con dieta sin sal, pobre en grasas, furosemida $40 \mathrm{mg} / 12$ horas, espironolactona $100 \mathrm{mg} / 24$ horas, enalapril $5 \mathrm{mg} / 24$ horas, digoxina y anticoagulantes orales.

Es valorada por nosotros en visitas a domicilio por desorientación con desconexión del medio que la paciente lo definía como "mente en blanco". Además refería angustia y temor. No presentaba cefalea, náuseas, vómitos, calambres musculares ni disminución de los reflejos. No tenía aumento de su disnea habitual ni edemas en MMII, ni dolor torácico. Le realizamos una analítica de urgencias objetivando un $\mathrm{K}$ : 5,2 mmol/1 y un $\mathrm{Na}$ : $110 \mathrm{mmol} / \mathrm{l}$ con el resto de los parámetros en los límites de la normalidad, enviándola al servicio de urgencias para tratamiento de su hiponatremia.

\section{DISCUSIÓN}

La utilización de los diuréticos en el tratamiento de múltiples patologías en Atención Primaria, nos obliga a conocer muy bien sus indicaciones así como sus posibles efectos secundarios.

La correcta utilización de los diuréticos por el Médico de Familia requerirá un conocimiento de la farmacología de cada grupo así como de la fisiopatología de la enfermedad de cada paciente.

En individuos sanos, con un filtrado glomerular normal, los riñones excretan en la orina el $1 \%$ del sodio filtrado, reabsorbiéndose, por tanto, el $99 \%$ del mismo. Es en el túbulo contorneado proximal donde se reabsorbe la mayor cantidad de sodio, un 60-70\%, en el asa de Henle lo hace el 20-30\%, en el túbulo contorneado distal (TCD) el $7 \%$ y en el tubo colector el $3 \%$ restante.

Cada diurético inhibe, por distintos mecanismos, la reabsorción de sodio en diferentes porciones de la nefrona, pudiendo llegar a inhibir casi por completo la reabsorción de sodio en dicha zona. Así los diuréticos de asa pueden excretar entre el 20-25\% del sodio filtrado, al inhibir la porción gruesa del asa de Henle; mientras que las tiazidas lo hacen en un 3-6\%, cuando bloquean la reabsorción en el TCD y los diuréticos ahorradores de potasio excretan el 1-2\% al inhibir competitivamente la acción de la aldosterona en el tubo colector ${ }^{4}$.

Centrándonos en el tratamiento diurético en la insuficiencia cardiaca por disfunción sistólica, hay que aclarar previamente que aunque los diuréticos son efectivos disminuyendo los síntomas de la congestión pulmonar y el edema periférico en los pacientes con insuficiencia cardiaca, no reducen la mortalidad $^{5-6}$.

Recogiendo las indicaciones de la American Heart Associaton y el American College of Cardio logy podemos decir que el tratamiento inicial de pacientes con insuficiencia cardiaca por disfunción sistólica debe comenzarse con los Inhibidores de la Enzima Convertidora de Angiotensina (IECAS), ya que en pacientes con fallo cardiaco medio, la reten- 
ción de sodio y el edema, no suele estar presente, y el uso de diuréticos no está indicado. Si comienza con ingurgitación yugular y edema periférico a pesar de la restricción de sal en la dieta, se deberá iniciar el tratamiento diurético con tiazidas; éstas deben usarse con sumo cuidado, requiriendo monitorización de los niveles de sodio al inicio del tratamiento en pacientes ancianos, y con antecedente de hiponatremia. Si aparece más sintomatología de insuficiencia cardiaca se le puede añadir un diurético de asa. Esta combinación requiere una cuidadosa monitorización del paciente para prevenir la depleción de volumen y una severa hipopotasemia.

La adición de espironolactona, a dosis diuréticas, para inhibir la reabsorción en el tubo colector cortical de sodio dependiente de aldosterona, puede aumentar ligeramente la excreción de sodio ${ }^{7}$.

Antes de la elección de un diurético de asa debemos tener en cuenta las siguientes cuestiones:
1. En pacientes con edema, la magnitud de la natriuresis y la diuresis inducida por diuréticos de asa, es mayor que en controles sanos ${ }^{3}$.

2. La furosemida tiene una absorción por vía oral muy errática, con un rango que oscila entre el 10 y el $100 \%$, y una vida media de 1,5 a 2 horas, mientras que la torasemida tiene un rango de absorción entre el 80 y el 100\%, con una vida media plasmática entre 3 y 4 horas. Estas características son clínicamente importantes ya que pacientes con insuficiencia cardiaca tratados con torasemida tendrán un menor número de reingresos y una mejor calidad de vida que los pacientes tratados con furosemida, a dosis equipotentes ${ }^{8}$.

3. Cuando el efecto diurético ha desaparecido, existe un aumento de los requerimientos de sodio por parte de los riñones, que, salvo restricción sódica en la dieta, contrarrestaría el efecto diurético ${ }^{5}$.

4. El sodio no reabsorbido en el asa de Henle, al pasar de forma crónica por el TCD, produce una

Tabla I

\begin{tabular}{|c|c|c|c|c|}
\hline \multicolumn{5}{|c|}{ MECANISM OS DE ACCIÓN, LUGARES DE ACCIÓN Y EFECTOS SECUNDARIOS DE LOS DIURÉTICOS ${ }^{11}$} \\
\hline Tipo de diurético & Sitio de acción & Mecanismo de acción & Hiponatremia & Otras complicaciones \\
\hline Diuréticos de Asa & $\begin{array}{l}\text { Rama ascendente } \\
\text { del asa de Henle }\end{array}$ & $\begin{array}{l}\text { cotransporte } \\
\mathrm{Na}-\mathrm{K}-2 \mathrm{Cl}\end{array}$ & $8 \%$ & $\begin{array}{l}\text { Hipopotasemia } \\
\text { Hipomagnesemia }\end{array}$ \\
\hline Diuréticos Tiazídic os & $\begin{array}{c}\text { Túbulo contorneado } \\
\text { distal }\end{array}$ & $\begin{array}{l}\text { Reabsorción } \\
\mathrm{Na}-\mathrm{Cl} \\
\text { electroneutra }\end{array}$ & $76 \%$ & $\begin{array}{l}\text { Hipopotasemia } \\
\text { Hipercalcemia } \\
\text { Hiperuricemia }\end{array}$ \\
\hline $\begin{array}{l}\text { Diuréticos } \\
\text { Ahorradores de potasio }\end{array}$ & $\begin{array}{l}\text { Túbulo distal y } \\
\text { tubo colector }\end{array}$ & $\begin{array}{c}\text { Intercambio de } \\
\mathrm{Na}-\mathrm{K} \text { por } \\
\text { inhibición de la aldosterona } \\
\text { o bloqueo de la entrada de } \mathrm{Na} \text {. }\end{array}$ & $1 \%$ & $\begin{array}{l}\text { Hiperpotasemia } \\
\text { Acidosis }\end{array}$ \\
\hline
\end{tabular}

Na: Sodio, K: Potasio, Cl: Cloro

Tabla II

FARMACOCINÉTICA DE LOS DIURÉTICOS

\begin{tabular}{lccc}
\hline Tipo de diuréticos & Biodisponibilidad (\%) & Vida media (h) & Comentarios \\
\hline Diuréticos de asa & & & \\
Furosemida & $10-100 \%$ & $0.3-3.4$ & Vida media aumenta en IR avanzada \\
Torasemida & $80-100 \%$ & $3-4$ & Vida media no varía en IR avanzada
\end{tabular}

\section{Diuréticos tiazídicos}

Clortalidona

Hidroclorotiazida

Indapamida

$\begin{array}{cc}65 \% & 24-55 \\ 65-75 \% & 3-10 \\ 90 \% & 6-15\end{array}$

Diuréticos ahorradores de potasio: Espironolactona
Duración del efecto: $24-72 \mathrm{~h}$

Duración del efecto: $6-12 \mathrm{~h}$

Duración del efecto: 24-36 h

Vida media de los metabolitos activos: $15 \mathrm{~h}$

IR: Insuficiencia Renal, h: horas 
hipertrofia de esta zona, aumentando el TCD su capacidad de reabsorber sodio. En pacientes que estén siendo tratados con un diurético de asa de manera crónica, la adición de un diurético tiazídico puede desencadenar una profunda diuresis al inhibir toda la reabsorción de sodio que se realiza en el $\mathrm{TCD}^{8}$.

En el estudio RALES (Randomized Aldactone Evaluation Study) realizado con pacientes en insuficiencia cardiaca por disfunción sistólica y una clase funcional III-IV de la NYHA, concluyeron que la asociación a un IECA y a un diurético de asa, de espironolactona a dosis bajas $(12,5 \mathrm{mg}$ a 50 mg por día) reducía la mortalidad por todas las causas, así como por causa cardiológica, disminuía la hospitalización por causa cardiaca, y mejoraba la clase funcional de los pacientes. Todo esto fue atribuido al efecto inhibitorio sobre la aldosterona de la espironolactona más que por el efecto diurético, ya que una dosis diaria de $25 \mathrm{mg}$ no tiene prácticamente efecto diurético, pero es suficiente para inhibir competitivamente la aldosterona. Con esta asociación, el número de efectos adversos no fue superior al del placebo'.

Muchas veces, en la práctica clínica diaria con pacientes en insuficiencia cardiaca, la toma de decisiones y el cambio terapéutico no resulta tan sencillo, ya que, como hemos comentado previamente, pequeñas variaciones en la dosificación de los diuréticos o en la ingesta hidrosalina pueden desencadenar profundos cambios en el equilibrio iónico de estos pacientes, produciéndose empeoramientos tanto por defecto (descompensación de su insuficiencia cardiaca) como por exceso (hiponatremia, hiperpotasemia, hipomagnesemia, deshidratación, etc.).

Uno de los efectos secundarios, que se pueden presentar por el uso de diuréticos en estos pacientes, es la hiponatremia. En una larga revisión de hiponatremias secundarias a tratamiento diurético, el $73 \%$ de los casos estaban producidos por diuréticos tiazídicos o clortalidona sola, un $6 \%$ por furosemida y sólo un $1 \%$ por espironolactona ${ }^{10}$. Las tiazidas, debido a sus características farmacológicas y farmacodinámicas, son el grupo diurético que más frecuentemente se relaciona con la hiponatremia. Las tiazidas no alteran la hipertonicidad medular, ya que actúan en el túbulo contorneado distal. La acción de la Hormona Anti Diurética
(ADH) en los túbulos colectores permitirá la reabsorción de agua hacia el intersticio hipertónico, viéndose incrementada la cantidad de agua libre reabsorbida, aumentando la tendencia a la hiponatremia (hipotónica). Además, en contraste con el resto de los diuréticos, las tiazidas tienen un efecto más prolongado y por tanto impiden la corrección de los déficits de volumen ${ }^{2}$.

Nuestra paciente no estaba en tratamiento con tiazidas, pero sí con furosemida y espironolactona que, a priori, tienen menos riesgo de producir hiponatremia que el tratamiento tiazídico. La combinación de un diurético de asa y espironolactona está recomendada tras los resultados del estudio RALES, aunque la elección de furosemida, como diurético de asa es discutida, y la dosis dada de espironolactona a nuestra paciente fuera superior a la recomendada por dicho estudio.

Ya hemos visto que cualquier diurético puede producir hiponatremia aunque sean las tiazidas las que con más frecuencia se relacionan con dicha complicación. Hay algunos estudios, sobre pacientes con insuficiencia cardiaca donde se sugieren otros mecanismos, igual de importantes, para la génesis de la hiponatremia, como el estímulo de la sed y el estímulo de la vasopresina que impide la excreción del agua libre, siendo especialmente problemático cuando los pacientes no cumplen con las restricciones en la ingesta de agua ${ }^{6}$.

Finalmente conviene señalar qué episodios de hiponatremia, en pacientes con insuficiencia cardiaca, se encuentran asociados con una menor supervivencia, sirviendo como marcador de enfermedad avanzada 6 .

CORRESPONDENCIA:

Julio Las Heras Mosteiro

C/ Moralzarzal, $61,5^{\circ} \mathrm{A}$

28034 Madrid

\section{Bibliografía}

1. Adrogue Horacio J, Madias Nicolaos E. Hyponatremia. $\mathrm{N}$ Eng J Med 2000; 342: 1581-9.

2. Rivera-Santos Angel, Star Robert A. Sodio, depleción de volumen, e hiponatremia. En: Donald Seldin,Gerhard Giebish. Agentes diuréticos: fisiología y farmacología. $1^{\mathrm{a}} \mathrm{ed}$. Madrid: Harcourt Brace 1999; 559-69. 
3. Gary Nicholls M. Interaction of diuretics and electrolytes in congestive heart failure. Am-J-Cardiol 1990; 65: 17E-21E.

4. Antes-LM, Fernandez-PC. Principles of diuretic therapy. Dis-Mon 1998 Jun; 44 (6): 254-68.

5. Rassol P. Treatment of edematous disorders with diuretics. Am J Med Sci 2000; 319 (1): 28-30.

6. Kramer Bernhard K, Schweda F, Riegger Günter AJ. Diuretic treatment and diuretic resistance in heart failure. Am J Med 1999; 106: 90-6.

7. Greenberg A. Diuretic complications. Am J Med Sci 2000; 319 (1): 10-24.
8. Craig Brater D. Diuretic therapy. N Engl J Med 1998; 339: 387-95.

9. Pitt B, Zannad F, Remme WJ, Cody R, Castaigne A, Perez A, Palensky J, et al. The effect of spironolactone on morbidity and mortality in patients with severe heart failure. N Engl J Med 1999; 341: 709-17.

10. Sonnenblick M, Friedlander Y, Rosin AJ. Diuretic induced sever hyponatremia. Review and analysis of 129 preported patients. Chest 1993; 103 601-6.

11. Craig Rater D. Pharmacology of Diuretics. Am J Med Sci 2000; 319 (1): 38-50. 